Address correspondence to: Doris Herzlinger, Cornell University Medical College, Physiology and Biophysics D-501, 1300 York Ave., New York, New York 10021-4805, USA. Phone: 212.746.6377; Fax: 212.746.8690; E-mail: daherzli@med.cornell.edu.

1. Johnson LR. Essential Medical Physiology. New York, New York, USA: Raven Press; 1992.

2. Becker A, Baum M. Obstructive uropathy. Early Hum Dev. 2006;82(1):15-22.

3. Hu P, et al. Ablation of uroplakin III gene results in small urothelial plaques, urothelial leakage, and vesicoureteral reflux. J Cell Biol. 2000;151(5):961-972.

4. Mendelsohn C. Using mouse models to understand normal and abnormal urogenital tract development. Organogenesis. 2009;5(1):306-314.

5. Airik R, Kispert A. Down the tube of obstructive nephropathies: the importance of tissue inter- actions during ureter development. Kidney Int. 2007;72(12):1459-1467.

6. Chang $\mathrm{CP}$, et al. Calcineurin is required in urinary tract mesenchyme for the development of the pyeloureteral peristaltic machinery J Clin Invest. 2004;113(7):1051-1058.

7. Santicioli P, Maggi CA. Myogenic and neurogenic factors in the control of pyeloureteral motility and ureteral peristalsis. Pharmacol Rev. 1998; 50(4):683-722.

8. Hurtado R, Bub G, Herzlinger D. The pelvis-kidney junction contains $\mathrm{HCN} 3$, a hyperpolarization-activated cation channel that triggers ureter peristalsis. Kidney Int. 2010;77(6):500-508.

9. Biel M, Wahl-Schott C, Michalakis S, Zong X. Hyperpolarization-activated cation channels: from genes to function. Physiol Rev. 2009;89(3):847-885.

10. David SG, Cebrian C, Vaughan ED Jr, Herzlinger D. c-kit and ureteral peristalsis. J Urol. 2005; 173(1):292-295.

11. Pezzone MA, et al. Identification of c-kit-positive cells in the mouse ureter: the interstitial cells of Cajal of the urinary tract. Am J Physiol Renal Physiol. 2003;284(5):F925-F929.

12. Lang RJ, Tonta MA, Zoltkowski BZ, Meeker WF, Wendt I, Parkington HC. Pyeloureteric peristalsis: role of atypical smooth muscle cells and interstitial cells of Cajal-like cells as pacemakers. J Physiol. 2006; 576(pt 3):695-705

13. Cain JE, Islam E, Haxho F, Blake J, Rosenblum ND. GLI3 repressor controls functional development of the mouse ureter. J Clin Invest. 2011; 121(3):1199-1206.

14. Yu J, Carroll TJ, McMahon AP. Sonic hedgehog regulates proliferation and differentiation of mesenchymal cells in the mouse metanephric kidney. Development. 2002;129(22):5301-5312.

15. Biesecker LG. What you can learn from one gene: GLI3. I Med Genet. 2006;43(6):465-469.

16. Riobo NA, Manning DR. Pathways of signal transduction employed by vertebrate Hedgehogs. Biochem J. 2007;403(3):369-379.

\title{
Unraveling virus-induced lymphomagenesis
}

\author{
Chris Boshoff
}

\author{
UCL Cancer Institute, University College London, London, United Kingdom.
}

\begin{abstract}
Kaposi sarcoma herpesvirus (KSHV), a human gammaherpesvirus, is the etiological agent for the endothelial-derived Kaposi sarcoma (KS) and also for certain lymphoproliferative disorders. In these lymphoproliferations, the KSHV-infected cells carry the stigmata of B lymphocytes, with plasmablastic features. The JCI has published three manuscripts addressing key questions related to $B$ cell infection and viral latent expression in B cells. Myoung and Ganem provide evidence that $\mathrm{CD}^{+}$lymphocytes suppress KSHV replication, promoting latency in B cells; Hassman and colleagues show that KSHV infection drives plasmablast differentiation in a subset of $\operatorname{IgM}^{+} \lambda$ light chain-expressing cells; and Ballon and colleagues describe the in vivo transdifferentiation of B lymphocytes by KSHV-encoded viral FLICE-inhibitory protein (vFLIP).
\end{abstract}

Two lymphotropic human herpesviruses are linked to lymphoma development: EBV and Kaposi sarcoma herpesvirus (KSHV). The mechanisms by which EBV infects B lymphocytes and induces their differentiation and proliferation are reasonably well understood (1). In vitro, EBV infection of human primary $B$ cells causes the establishment of latent infection in a fraction of cells exposed to virus, cellular transformation, and the outgrowth of indefinitely proliferating B lymphoblastoid cell lines. In contrast, the lack of B cell systems available for the study of KSHV in vitro and in vivo has hampered our understanding of the natural life cycle of KSHV in B cells and of KSHVinduced B cell lymphoproliferations. The

Conflict of interest: The author has declared that no conflict of interest exists.

Citation for this article: J Clin Invest. 2011; 121(3):838-841. doi:10.1172/JCI46499.
$J C I$ has now published three papers (2-4) that reveal provocative findings regarding KSHV and B cell infection and function.

The main route for infection by EBV and KSHV is via saliva. EBV enters tonsillar B cells via the CD21 receptor and steers the differentiation of pregerminal naive B lymphocytes toward memory cells by way KSHV in saliva (5) and in tonsillar and peripheral CD19+ B cells (6) and the inefficient in vitro infection of primary nonstimulated B lymphocytes from PBMCs prompted the groups of Don Ganem (2) and Dean Kedes (3) to utilize primary tonsillar explants to study KSHV infection ex vivo. Previously, efficient productive or lytic infection of IL-4 and CD40 ligand-activated PBMC-derived B lymphocytes and infection of B lymphocytes from tonsils were demonstrated (7). It is unclear whether activation of B lymphocytes results in the of viral latent transcripts. The presence of upregulation of surface molecules required for KSHV infection, for example, heparin sulfate (8) and DC-SIGN (CD209) (7), and/or whether such activation triggers signaling pathways that encourage viral entry and intracellular transport (9).

Myoung and Ganem showed that exposure of primary human tonsillar explants to KSHV virions results in infection of $\mathrm{B}$ and $\mathrm{T}$ lymphocytes, with B lymphocytes producing substantial amounts of infectious virions (2). Strikingly, and in contrast to exposure of B lymphocytes to EBV, KSHV displays predominantly lytic infection in tonsillar-derived B lymphocytes. This spontaneous lytic viral reactivation of infected B lymphocytes was suppressed when the investigators added activated $\mathrm{T}$ lymphocytes from tonsillar explants. However, these activated $\mathrm{CD} 4^{+} \mathrm{T}$ lymphocytes did not induce B lymphocyte cytolysis and were not dependent on autologous $\mathrm{T}$ lymphocytes being used. Thus, the suppression of spontaneous viral lytic cycle entry in B lymphocytes was MHC unrestricted and not dependent on killing of target cells. Treatment of mixed cultures with the $\mathrm{T}$ cell inhibitor, cyclosporine, abrogated the inhibition of lytic replication. Myoung and Ganem found that activated viable $T$ lymphocytes require physical contact with the infected B lymphocytes to inhibit lytic virus replication. They therefore proposed that unidentified effector $\mathrm{T}$ cell surface ligands are responsible for $\mathrm{T}$ cell-target cell 
A

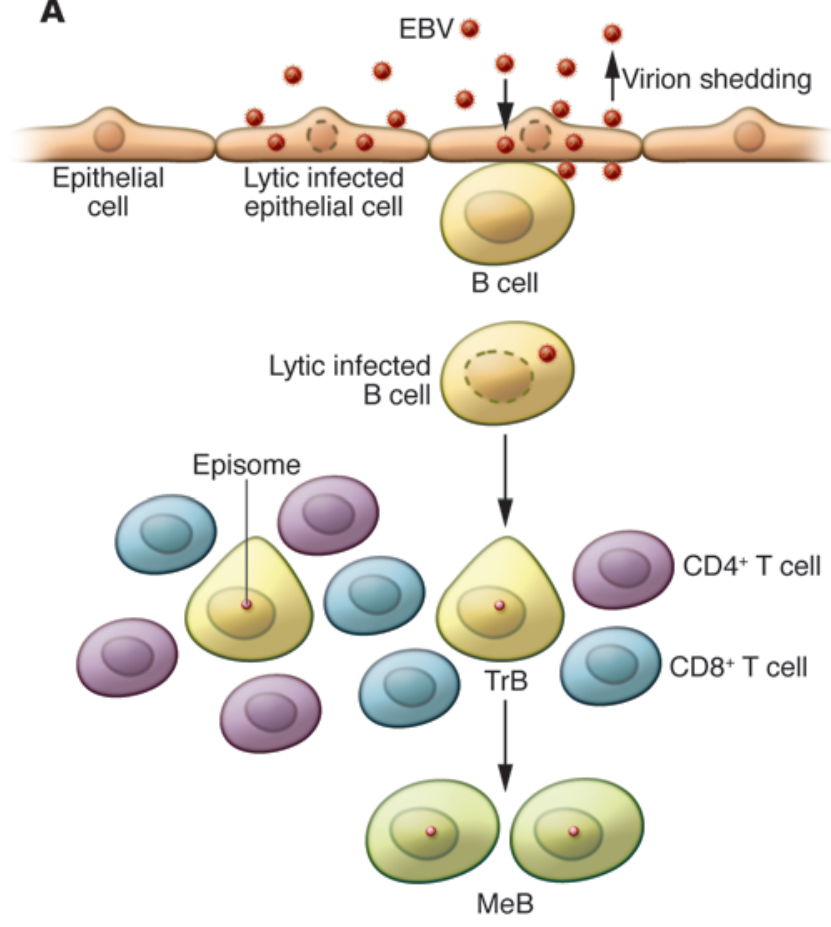

B

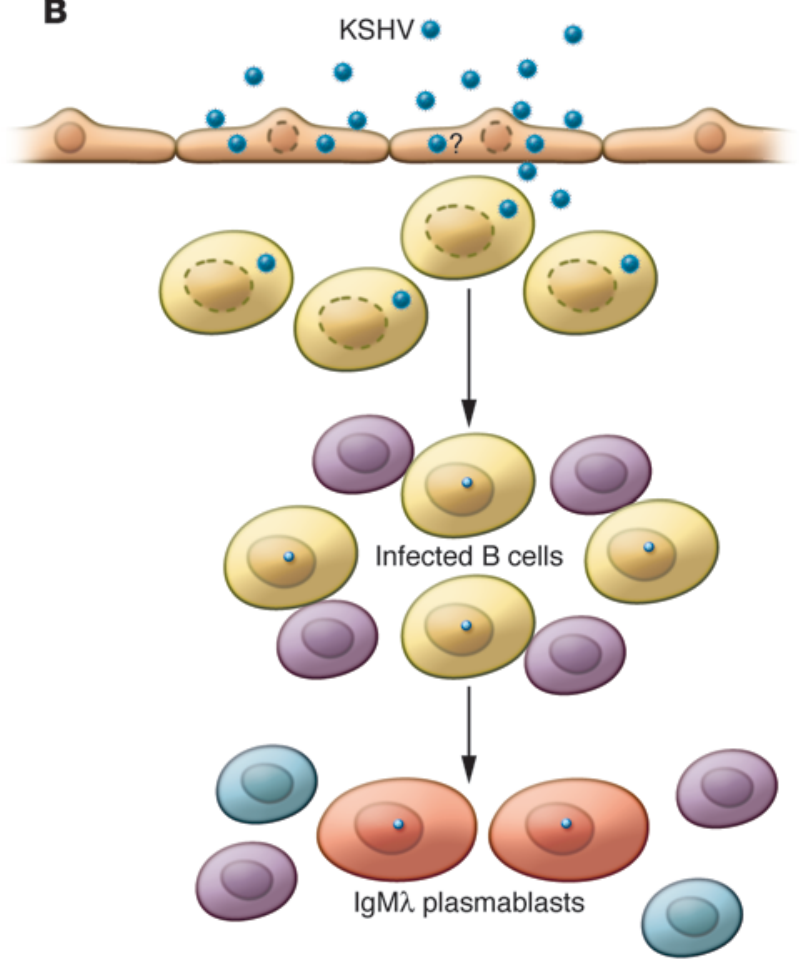

Figure 1

Early events after EBV and KSHV infection of tonsillar cells. (A) EBV is amplified by permissive epithelial cells (lytic infection) and infects mucosal naive B cells. The viral default pathway in B cells is latent infection, where EBV persists as an episome (red circle). A minority of infected $B$ cells are transformed (TrB). In infectious mononucleosis, a significant expansion of transformed lymphoblastoid cells occurs. Anti-EBV antigen $\mathrm{CD} 4^{+}$and $\mathrm{CD} 8^{+} \mathrm{T}$ cells control the proliferation of transformed cells. EBV persists in B lymphocytes, as part of the long-lived memory B cell pool (MeB). (B) Early events during KSHV infection are less established. It is uncertain whether B cells become infected after amplification of KSHV in epithelium. Data presented in this issue suggest that KSHV induces significant spontaneous lytic replication ex vivo in tonsillar-derived B cells (2). This lytic infection is suppressed when infected B cells come in contact with activated CD4+ $T$ cells. The suppression of spontaneous viral lytic cycle entry in B cells is MHC unrestricted and not dependent on killing of target cells. New data also suggest that the primary target for KSHV in tonsillar explants could be $\mathrm{IgM}^{+}$memory B cells (3). The majority of KSHV latent infected B cells after exposure of tonsillar explants to virus express $\lg M \lambda$. KSHV induces plasmablastic differentiation of these $\lg M \lambda^{+}$cells. An enrichment of IgM $\lambda$-expressing plasmablasts also occurs in inducible vFLIP knockin mice, targeting vFLIP to different stages of B cell proliferation (4). Such IgM $\lambda+$ infected plasmablasts are thought to cause KSHV-related MCD. Lytic infected cells are indicated by disrupted nuclear membranes.

recognition and might trigger an exocytosis event in the effector T cells, releasing factors to the KSHV-infected B lymphocytes.

These in vitro findings contrast with what we have learned about primary EBV infection (10): the current paradigm is that lack of functional $\mathrm{T}$ lymphocytes, for example, induced by iatrogenic or acquired immunosuppression, leads to the in vivo outgrowth of latent infected B lymphocytes and subsequent EBV-driven lymphoproliferations such as posttransplant lymphoproliferative disease. Myoung and Ganem propose that $\mathrm{T}$ lymphocyte activation is necessary to block KSHV lytic reactivation in B lymphocytes, promoting latent infection (Figure 1).

It is unclear whether B lymphocytes display de novo entry into the lytic cycle (i.e., the lytic cycle being the default pathway) or whether high level spontaneous reac- tivation occurs from latency. It will be of significant interest to know whether these intriguing findings occur in vivo and whether this suppression of lytic reactivation is restricted to primary infection within the tonsillar microenvironment.

Prior to the introduction of effective antiHIV treatment, it was noted that nearly 50\% of those who acquired KSHV after HIV infection went on to develop Kaposi sarcoma (KS). Thus, being exposed to KSHV in the setting of a damaged $\mathrm{T}$ lymphocyte immune response would result in a higher KSHV viral load set point and an increased risk of developing KS. The findings by Myoung and Ganem could help explain this observation: a diminished $\mathrm{T}$ lymphocyte response upon primary KSHV infection would result in enhanced spontaneous B lymphocyte reactivation and a higher viral load.

\section{KSHV and plasma cell disorders}

B cell neoplasia associated with KSHV infection includes the monoclonal primary effusion lymphoma (PEL) (11) and a plasmablastic variant of multicentric Castleman disease (MCD) (12), a polyclonal neoplasm. In both these tumors, KSHV infection is associated with preterminally differentiated plasma cells with either cytoplasmic or surface immunoglobulins. Mature B cells exhibit allelic exclusion in which only a single class of Ig heavy chain and a single class of light chain, $\kappa$ or $\lambda$, is expressed. Light chain restriction in a $\mathrm{B}$ cell population is usually considered proof of monoclonality and therefore cancer. However, KSHV-infected plasmablasts in MCD are an exception, in which polyclonal expansion of $\operatorname{IgM} \lambda$ light chainexpressing KSHV-infected plasmablasts is the hallmark (refs. 13-15 and Figure 2). 
A

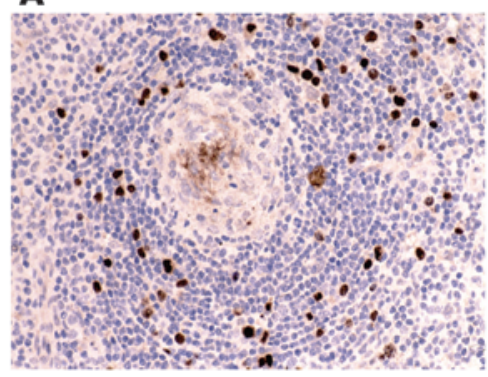

B

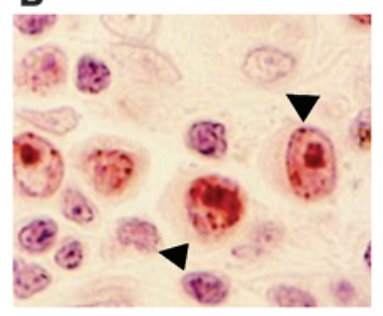

Figure 2

KSHV-related plasmablastic MCD. (A) KSHV-LANA-positive cells are scattered throughout the mantle zone of a lymph node affected by MCD. (B) Higher magnification of MCD mantle zone. The KSHV-positive cells (black arrowheads) are large, have prominent nuclei with 1 or 2 nucleoli, and resemble plasmablastic cells. Lymphocytes surrounding the plasmablasts stain negative for KSHV. Reprinted with permission from ref. 13 (copyright 1999, National Academy of Sciences, USA). Original magnification, $\times 140$.

This is a curiosity, as almost all other lymphoproliferative disorders demonstrate both $\kappa$ and $\lambda$ light chain restriction, and no functional difference between these light chains is identified. This observation suggests that KSHV either preferentially infects $\lambda$ light chain-expressing lymphocytes, that $\lambda$ light chain cells provide a survival and/or proliferation advantage to KSHV-infected B cells, or that KSHV preferentially steers expansion of $\lambda$-expressing cells.

The Kedes group investigated whether KSHV preferentially infects plasmablasts or infects a less differentiated cell and actively drives them toward a plasmablast phenotype (3). In a series of elegant experiments, Hassman et al. employed multispectral imaging flow cytometry, which combines the high-throughput power of flow cytometry with the morphological and subcellular spatial detail of multicolor fluorescent imaging to identify and characterize KSHVinfected cells. First they demonstrated that within ex vivo suspensions of human tonsillar cells, KSHV infection (as measured by cells expressing the latency-associated nuclear antigen [LANA]) preferentially occurred in B cells. Even though both light chain subsets were present in the tonsillar cultures in comparable proportions, LANA-positive cells were almost exclusively observed in the $\lambda$ subset. They show further that KSHV infection drives the proliferation of IgM $\lambda$ tonsillar B cells. In addition, infected cells acquired phenotypic changes mimicking MCD plasmablasts, including the blasting morphology, IgM expression, and high levels of IL-6 receptor expression.

Hassman et al. raise an intriguing, but plausible possibility: rather than targeting naive B cells, KSHV preferentially infects and drives the proliferation of $\operatorname{IgM}^{+}$memory B cells. Such cells are present in tonsils and spleen and become $\mathrm{IgM}^{\mathrm{hi}}, \mathrm{Ki}^{+} 7^{+}$, and $\mathrm{CD} 27^{+}$ during plasmablast differentiation (16). Such differentiation might be triggered by KSHV-induced NF- $\kappa$ B activation (see below), and such a phenotype is compatible with the plasmablasts present in their infected tonsillar cultures and in MCD.

\section{NF- $\kappa B$ and $B$ cell plasticity}

Similar to other herpesviruses, only a fraction of KSHV open reading frames are expressed during latency, with the majority being expressed when the virus is triggered to enter the lytic program, resulting in cells exuding infectious virions. Among these latent viral proteins is the viral FADD-like IL-1 $\beta$-converting enzyme (FLICE/caspase 8) inhibitory protein (vFLIP). Whereas cellular FLIP proteins associate with FADD/DISC, preventing caspase-8-induced apoptosis, vFLIP in KSHV-infected cells, including PEL cells, is mainly associated with the IкB kinase (IKK) complex, leading to I $\mathrm{B} \alpha \alpha$ degradation, followed by release of NF- $\kappa \mathrm{B}(17,18)$. Activation of NF- $\mathrm{\kappa B}$ is a mechanism exploited by lymphomagenic viruses to prolong cellular survival and to induce proliferation. EBV and human T cell leukemia virus 1 (HTLV-1) encode viral oncoproteins, LMP1 and Tax, constitutively activating NF- $\mathrm{KB}(19,20)$.

The Cesarman group generated inducible vFLIP knockin mice, targeting vFLIP to different stages of $\mathrm{B}$ cell proliferation (4). The activation of transgene expression was achieved by crossing the vFLIP knockin mice with mice expressing cre recombinase under the control of either the CD19 or C $\gamma 1$ promoter, resulting in vFLIP expression in all $\mathrm{CD} 19^{+} \mathrm{B}$ cells or more restrictedly, in IgG1+ germinal center (GC) B cells. Within three months, TG mice developed splenomegaly with erased GCs and defective Ig class switch recombination (CSR) and affinity maturation. Within 20 months, most TG animals developed histiocytic/DC sarcoma of B cell origin.

Although these vFLIP TG mice did not develop tumors faithfully mimicking either human MCD or PEL, a number of striking findings were made. First, an enrichment of IgM $\lambda$-expressing plasmablasts occurred in all mice. The findings in these transgenic mice support the conclusion that KSHV latent transcripts, including vFLIP, are responsible for the preferential expansion of $\operatorname{IgM} \lambda^{+}$cells. This is supported by the findings of Hassman et al., who showed that KSHV selectively established latent $\left(\mathrm{LANA}^{+}\right)$infection almost exclusively in Ig $\lambda$ B cells, despite the fact that both Igא and $\operatorname{Ig} \lambda$ B lymphocytes expressed the lytic viral protein PAN after exposure of CD $19^{+}$ lymphocytes to KSHV (3), and by the discovery that $\lambda$-chain-positive $B$ cell development is dependent on NF-кB (21). Second, the Ballon et al. study reveals the in vivo immunological consequences of vFLIP when expressed in B cells. The abrogation of GC formation and inhibition of CSR and affinity maturation by vFLIP could directly contribute to KSHV pathogenesis by curtailing host immunity. Third, this work indicates the in vivo capacity of vFLIP to reprogram/transdifferentiate B cells into histiocytes. This finding supports the clonal B cell origin of histiocytic sarcoma, which can develop in individuals with $B$ cell lymphoproliferations (22), including KSHV-related MCD, and argues that NF-кB signaling contributes directly to the in vivo plasticity of B cells.

\section{Future directions}

These three studies are sound foundations for further investigations of KSHV infection in vivo. Humanized mouse models could be used to test the in vivo relevance of the studies by Myoung et al. and Hassman et al. and to decipher the molecular mechanisms favoring IgM $\lambda$ B lymphocyte expansion and $\mathrm{B}$ cell transdifferentiation $(2,3,23)$. Furthermore, the vFLIP transgenic mice described by Ballon et al. could be crossed with mice expressing other KSHV latencyassociated transcripts and complement the development of inducible TG mice expressing more than one latent transcript (3). We 
could also speculate that the activation of vFLIP (with or without other latent viral transcripts) at a more mature stage of B cell differentiation, such as in post-GC B cells, will permit completion of the $\mathrm{GC}$ reaction and better recapitulate KSHV-lymphoproliferation development. These three studies are opening up new avenues to explore the immunobiology of KSHV as it relates to its principal reservoir, B lymphocytes.

\section{Acknowledgments}

Chris Boshoff is supported by Cancer Research UK, the Medical Research Council, and the UCL/University College London Hospital Comprehensive Biomedical Research Centre.

Address correspondence to: Chris Boshoff, UCL Cancer Institute, University College London, London, United Kingdom, WC1E 6BT. Phone: 44.20.7679.6850; Fax: 44.20.7679.6817; E-mail: c.boshoff@ ucl.ac.uk.

1. Alfieri C, Birkenbach M, Kieff E. Early events in Epstein-Barr virus infection of human B lymphocytes. Virology. 1991;181(2):595-608.

2. Myoung J, Ganem D. Active lytic infection of human primary tonsillar B cells by KSHV and its noncytolytic control by activated CD4 ${ }^{+} \mathrm{T}$ cells. J Clin Invest. 2011;121(3):1130-1140

3. Hassman LM, Ellison TJ, Kedes DH. KSHV infects a subset of human tonsillar B cells, driving proliferation and plasmablast differentiation. J Clin Invest. 2011;121(2):752-768.

4. Ballon G, Chen K, Perez R, Tam W, Cesarman E. Kaposi sarcoma herpesvirus (KSHV) vFLIP oncoprotein induces B cell transdifferentiation and tumorigenesis in mice. J Clin Invest. 2011; 121(3):1141-1153.

5. Pauk J, et al. Mucosal shedding of human herpesvirus 8 in men. NEngl J Med. 2000;343(19):1369-1377.

6. Ambroziak JA, et al. Herpes-like sequences in HIVinfected and uninfected Kaposi's sarcoma patients. Science. 1995;268(5210):582-583.

7. Rappocciolo G, et al. Human herpesvirus 8 infects and replicates in primary cultures of activated B lymphocytes through DC-SIGN. J Virol. 2008; 82(10):4793-4806.

8. Birkmann A, et al. Cell surface heparan sulfate is a receptor for human herpesvirus 8 and interacts with envelope glycoprotein K8.1. J Virol. 2001; 75(23):11583-11593

9. Chandran B. Early events in Kaposi's sarcoma-associated herpesvirus infection of target cells. J Virol. 2010;84(5):2188-2199.

10. Hislop AD, Taylor GS, Sauce D, Rickinson AB. Cellular responses to viral infection in humans: lessons from Epstein-Barr virus. Annu Rev Immunol. 2007;25:587-617.

11. Cesarman E, Chang Y, Moore PS, Said JW, Knowles DM. Kaposi's sarcoma-associated herpesvirus-like DNA sequences in AIDS-related body-cavity-based lymphomas. N Engl J Med. 1995;332(18):1186-1191.

12. Soulier J, et al. Kaposi's sarcoma-associated herpesvirus-like DNA sequences in multicentric Castleman's disease. Blood. 1995;86(4):1276-1280.

13. Dupin N, et al. Distribution of human herpesvirus-8 latently infected cells in Kaposi's sarcoma, multicentric Castleman's disease, and primary effusion lymphoma. Proc Natl Acad Sci U S A. 1999; 96(8):4546-4551.

14. Dupin N, et al. HHV-8 is associated with a plasma- blastic variant of Castleman disease that is linked to HHV-8-positive plasmablastic lymphoma. Blood. 2000;95(4):1406-1412.

15. Du MQ, et al. Kaposi sarcoma-associated herpesvirus infects monotypic (IgM lambda) but polyclonal naive B cells in Castleman disease and associated lymphoproliferative disorders. Blood. 2001;97(7):2130-2136.

16. Kurosaki T, Aiba Y, Kometani K, Moriyama S, Takahashi Y. Unique properties of memory B cells of different isotypes. Immunol Rev. 2010;237(1):104-116.

17. Liu L, Eby MT, Rathore N, Sinha SK, Kumar A, Chaudhary PM. The human herpes virus 8-encoded viral FLICE inhibitory protein physically associates with and persistently activates the Ikappa B kinase complex. J Biol Chem. 2002;277(16):13745-13751.

18. Field N, et al. KSHV vFLIP binds to IKK-gamma to activate IKK. J Cell Sci. 2003;116(pt 18):3721-3728.

19. Laherty CD, Hu HM, Opipari AW, Wang F, Dixit VM. The Epstein-Barr virus LMP1 gene product induces A20 zinc finger protein expression by activating nuclear factor kappa B. J Biol Chem. 1992;267(34):24157-24160.

20. Suzuki T, Hirai H, Fujisawa J, Fujita T, Yoshida M. A trans-activator Tax of human T-cell leukemia virus type 1 binds to NF-kappa B p50 and serum response factor (SRF) and associates with enhancer DNAs of the NF-kappa B site and CArG box. Oncogene. 1993;8(9):2391-2397.

21. Derudder E, et al. Development of immunoglobulin lambda-chain-positive B cells, but not editing of immunoglobulin kappa-chain, depends on NFkappaB signals. Nat Immunol. 2009;10(6):647-654.

22. Feldman AL, et al. Clonally related follicular lymphomas and histiocytic/dendritic cell sarcomas: evidence for transdifferentiation of the follicular lymphoma clone. Blood. 2008;111(12):5433-5439.

23. Ma SD, et al. A new model of Epstein-Barr virus infection reveals an important role for early lytic viral protein expression in the development of lymphomas. J Virol. 2011;85(1):165-177.

\title{
Adherent-invasive E. coli in Crohn disease: bacterial "agent provocateur"
}

\author{
Warren Strober
}

\begin{abstract}
Mucosal Immunity Section, Laboratory of Host Defenses, National Institute of Allergy and Infectious Diseases (NIAID), NIH, Bethesda, Maryland, USA.
\end{abstract}

\begin{abstract}
The role of adherent-invasive E. coli (AIEC) in Crohn disease (CD) has been in debate for decades. AIEC bacteria are found in the small intestine of patients with chronic $C D$, but it has remained unclear whether this infection is causal or secondary to underlying immune deficiencies in $C D$ patients. In this issue of the JCI, Chassaing and colleagues demonstrate that AIEC bacteria express an adherence factor called long polar fimbriae (LPF) that aids in the binding of these bacteria to $M$ cells overlying Peyer's patches and subsequent entry into lymphoid tissue. These findings provide a mechanism of AIEC penetration but do not prove that AIEC is causing a primary infection in the Peyer's patches that is necessary for the initiation or persistence of CD inflammation.
\end{abstract}

Conflict of interest: The author has declared that no conflict of interest exists.

Citation for this article: J Clin Invest. 2011;

121(3):841-844. doi:10.1172/JCI46333.
Invasive E. coli as a cause of Crohn disease: a trail of research

The concept that Crohn disease (CD) is due to an infectious organism has been under investigation since this inflammation was first distinguished from mycobacterial infection some 80 years ago. While in recent decades enthusiasm for this concept has waned in the face of evidence that the disease is due to a dysregulated (and excessive) immune response against one or more commensal organisms in the intestinal microflora, creditable research is still being conducted to establish its validity. By far the most impressive example of this work is that of Darfeuille-Michaud and colleagues, who have accumulated a large body of data showing that an E. coli organism may be involved in $\mathrm{CD}$ pathogenesis. The basic findings of these investigators are as fol- 